$\therefore \quad 1$

For Internal Distribution Only

Accelerator Division

Alternating Gradient Synchrotron Department

BROOKHAVEN NATIONAL LABORATORY

Upton, New York 11973

Accelerator Division

Technical Note

AGS/AD/Tech. Note No. 433

BNL MAD Program Notes

Tracking Plots

J. Niederer

April 19, 1996 


\title{
BNL MAD Program Notes
}

\author{
Tracking Plots \\ J. Niederer \\ AGS Department \\ Brookhaven National Laboratory
}

April 19, 1996

\section{Overview}

The ancient BNL MAD track plotting service is being upgraded, at least partially to support AGS extraction studies. One extension is to display phase plots at more than one place along an orbit. A second extension offers menu based controls that allow a viewer to watch a phase plot build up, turn by turn, place by place, or track by track. A third is a fairly elaborate track pattern generating utility.

Historically this kind of plotting has been done off line using an intermediate file that receives computed track coordinates, which is then input to harmonic analysis and plotting commands. For the present the plotting has been substantially revised for use with modern graphics workstations, and improved with various menu selection features. Perhaps a further approach would be to attach similar plotting on line to the tracking calculations, along with utilities that allow various machine parameters to be changed during the tracking, as is now available with the "Twiss" closed orbit and neural feedback screen graphics managers. Less visible, the internal file system services have been rewritten to remove ten year old compromises involving certain vendor file handling idiosyncracies, particularly at CERN, and fix a number of other annoying problems. Working defaults for all of the file descriptions and handling are provided in the recent versions of dictionaries, so these complications are generally transparent to the program user. In principle the file handling and graphics are portable with $\mathrm{X}$ Windows versions, give or take remaining vendor software difficulties.

\section{Menu Options}

Track plotting typically involves scatter plots among transverse position coordinates $\mathrm{X}$ or $\mathrm{Y}$ and direction coordinates Px or Py, or quantities derived from them, as described in the MAD Users Manual. In extraction studies, phase plots of $\mathrm{X}$ vs Px are often used to study beam behavior as the beam is perturbed. The discussion here will consider phase plots, but applies equally well to beam spots in $\mathrm{X}$ and $\mathrm{Y}$, or other combinations. In circular machines, such plots might give the history of a particle over many turns, and the resulting clutter may hide the final behavior as the orbit is perturbed. The newer menu options allow a viewer to follow a particle over a set of time and position steps. These features apply to the conventional MAD Single, and Multiple plot types, and to the newer Ellipse plot type. For the single type, the phase history of a each particle is plotted on a separate picture. For the multiple type, the phase histories of all included tracks are plotted on the same picture, which can get confusing if the initial complement of tracks is random. For the ellipse type, the histories of one or more generated phase ellipses are drawn. Older versions of MAD presumed a single observation point for recording track coordinates; the current one permits as many as patience and the file system can handle.

\subsection{Menu for Single and Multiple Types}

When the menu option is selected on the plotting command, a popup menu presents a number of choices for advancing one or more tracks, and selecting among tracks and observation places. Selections are made by depressing the right mouse button. The menu can be "dragged" about on the screen by putting 
the cursor over the top (label) panel, depressing the right mouse button, and moving the cursor with the button down. The program scans the track history file for all entries (events) for a given place and track, then advances to the next place if any, scans again, and continues through the places. It then repeats the scans for the next track if any, cycling through the places again. The viewer can in effect step the drawing event by event, place by place, and track by track.

The first three menu panels display:

1. The type of plot, single or multiple.

2. The current place index. (Observation Point)

3. The current track index.

The remaining menu panels offer the choices:

Do 1 Draw a single track point, and return to the menu. A track point is the set of coordinates of a particle track at a particular place and turn (time).

Do 5 Draw five successive track points, or less if at the end of a place, and return to the menu. Each such point occurs at a succeeding turn, or multiple of turns, depending on the recording and the sampling criteria.

Do 10 Draw ten successive track points, or less if at the end of a place, and return to the menu.

Do 25 Draw 25 successive track points, or less if at the end of a place, and return to the menu.

Do Place Drawing continues until all remaining track points for the current place and track have been treated.

Do Track Drawing continues until all remaining track points, and places, for the current track have been treated.

Do All Drawing continues until all remaining track points, and places, and tracks have been treated. This option corresponds to the usual way drawing is done, and gives an overview for further viewing.

Next Place Indexing advances without drawing to the next place, or to the next track and the first place.

Next Track Indexing advances without drawing to the next track, or to the second, end of drawing menu.

Clear The central region of the drawing holding phase or other points is cleared, and the frame redrawn.

Repeat Control returns to the start of the cycling, positioned at the first entry, first place, and first track.

Quit The picture is cleared and the program exits from the plot section, to the next command on the input file.

In Single plot mode, the program stops after all entries for a track have been finished, and writes "Pause" in the window from which the program is run. Hitting the Enter key continues the plotting, bringing up the menu in menu mode, and otherwise drawing the next track or finishing.

In menu mode, when the various cycles of tracks and places have finished, a second menu is presented:

Single The plot type is set to Single, and control returns to the same menu, allowing other choices to be changed, before repeating.

Multiple The plot type is set to Multiple, and control returns to the same menu.

Set to X, Px The plotting variables are set to X for the Horizontal, and Px for the Vertical, and control returns to the same menu.

Set to Y, Py The plotting variables are set to Y for the Horizontal, and Py for the Vertical, and control returns to the same menu. 
Repeat The drawing process with any changes entered above can be repeated.

Quit The program removes the drawing window and leaves the plotting section.

\subsection{Menu for Ellipse Type}

The ellipse type of plot is normally intended for one or more some small groups of tracks, originally generated from X - Px and Y - Py phase ellipses. Instead of single tracks, coordinate histories of groups of related tracks are of interest. Menu choices thus reflect data for a group, a pattern of groups at a place, and a pattern of places at succeeding turns. Menu items are merely listed here, as they have the same or similar meanings to the list in Section 2.1 above.

The first three (inactive) menu panels display indices for:

1. The current group index.

2. The current place index.

3. The current turn index.

The active menu panels are:

Do Group

Do Place

Do Turn

Do All

Next Group

Next Place

Next Turn

Clear

Repeat

Quit

An end of sequence menu provides for repeating in the X - Px or Y - Py Plane:

Set to X, Px This choice changes the variables, and returns to the first ellipse menu, like Repeat.

Set to Y, Py This choice changes the variables, and also returns to the first ellipse menu.

Repeat

Quit

\section{The FTrplot Command}

The BNL MAD track plotting section, upgraded from the older MAD PLOT command, has been augmented to deal with workstation graphics, menus, screen plotting symbols, and drawing phase ellipses. Each change involves one or more additional parameters given below. The original set of parameters described in the MAD Users Manual have been retained. The PItrplot command is an equivalent version of FTrplot, but keyed to the graphics package, and intended for future use in picture lists of drawing commands.

Track plotting obtains its information from a so called MAD tracking History File, produced by the Frun (Run) command. If not told otherwise, the program will produce an unformatted direct access file called \$STORY.01, for the tracking history, which remains in the directory. Tracking may be rather slow compared with the displays, but on the other hand a number of trials may be needed to get the display parameters suitably developed. Hence, if a lengthy tracking run is involved, it may be worthwhile to archive these results:

\section{Archive "*STORY.01"}

which produces a second file called_STORY.01 Internal name characters such as "**" which are troublesome to file directories are changed when files are written. These names need to be enclosed in quotes as 
special characters are involved, mostly to avoid accidental name conflicts within the data base. The results can be recalled for repeated plotting operations by:

Retrieve "*STORY.01"

\subsection{Original FTrplot Attributes}

Bank The name of the history (bank) table. Default is *STORY.01

Haxis A name drawn from among choices of X, Px, Y, Py, Dt, Deltap, Wx, Wy, Wt, Turns, Xn, Pxn, Yn, Pyn, Phix, Phiy, Phis: the variable to be plotted along horizontal axis.

Vaxis A name from the Haxis list above: the variable to be plotted along vertical axis.

Hscale(2) Optional value for half width of horizontal scale. Otherwise the program will do its own scaling. Self scaling will be considerably distorted by tracks leaving from large orbits.

Vscale(2) Optional value for half width of vertical scale.

Type Optional Name of Plot Type: "Single", "Multiple", "Scatter", or "Ellipse"

Freq Integer value of sampling frequency of turns for plotting. Multiplies any previous turn sampling on history file. Default is 1.

In the newer edition, if any second scale entries are given, plotting is done for values lying between the two entries, which can help to make better use of the limited display space.

\subsection{Ellipse Additions}

Phase ellipses may be drawn corresponding to the track groups prepared by a FTrgen command, or suitable collection of Start commands. Unlike the scatter plots produced by the other Type options, the Ellipse option produces closed curves by joining the tracks originally generated along one or more phase ellipses. A set of these ellipses may be centered according to some spatial pattern. This feature is intended for displaying families(groups) of particle tracks, for which members share some common trait, such as off momentum. One or more such families are tracked for a single pass, and phase coordinates are recorded for each at one or more places along the orbit. One or more equal sized groups make up the total number of tracks being followed. A typical application is tracking through an irregular magnetic field, or beam line.

Colors and line types are taken from vector arrays attached to a Pendata command in the plot description file. Pen (line) characteristics are indexed first to the track groups, and second to the ordered positions at which phase data are recorded. To set up colors and line styles (solid, dots, dashes, etc), for a sample of three places, the color and style arrays should step as:

$$
\begin{aligned}
& \text { Tr_col Mvector } \mathrm{N} 1=30,1,1,1,2,2,2,3,3,3, \ldots \\
& \text { Tr_stl Mvector } \mathrm{N} 1=30,0,11,12,0,11,12,0,11,12, \ldots \\
& \text { Tr_pen Pendata Color }=\mathrm{Tr}_{\text {_col, }} \text { Style }=\mathrm{Tr} \text { _stl, Width }=\mathrm{Tr} \text { _wid, .... }
\end{aligned}
$$

This pattern will color an ellipse red, green, yellow, etc, according to track group, and use solid, dotted, or dashed joining lines according to observation place.

The FTrplot command has the additional Type option of "Ellipse", which causes the drawing of closed ellipses at selected points along a lattice. This option is keyed to the starting track ensemble generated by FTrgen or FStart commands for starting tracks. When using the ellipse features, a Print command should be used to note all lattice points for which track data is to be recorded. The Frun command parameters Turns and Fprint and Sample should be set with some awareness about the often large amount of data which will appear on the pictures.

The additions to the Plot command as described in the MAD V7 Users Manual are: 
Type The Ellipse option has been added to the list of types of plot.

Join Logical Flag: If True, the points from each track group will be joined by a line. Joining is done automatically for a track family generated as points on a phase ellipse. The drawing of outlines among track points can often help to show the way orbits distort with time.

\subsection{Workstation Plot Description}

Workstation screen plotting involves a lengthy set of description commands, usually provided as a separate input statement file with the program. The alternative of hard wiring within the program the immense amount of detail even down to the level of colors is worse. These details are contained in a set of commands loosely organized under a BNL MAD Plotdef command. The details can be customized to taste by adventurous users. Plotting has been designed to work with a plotting package based on the object oriented MAD data base, and the Silicon Graphics dedicated Graphics Library (GL) screen display primitives. A locally written X Windows version of the GL sections used in BNL MAD hopefully makes these graphics additions portable, beyond Silicon Graphics screens.

Plotdef The name of a Plotdef command. To obtain screen plotting, either a customized plot file or the default plot file must be available in the input data. A complete plot setup is available as a default, which requires the plot definition CALL file Trplots.call to be available as part of the input data.

Menu Logical Flag: If True, and a Plotdef is given, drawing will be operated from a menu, as described above.

Prplot Logical Flag: If True, an archaic form of printer plot will be attempted. This feature has been benignly neglected.

Hard copy from screen graphics is currently obtained with the Silicon Graphics "snapshot" utility, which produces a screen dump over a selected area. This dump file can be converted to a color or ordinary Post Script file by the "tocolps" ("tops") utility, or massaged by Xview or similar utilities. The resolution is only about 100 dots per inch, not a particularly good match to modern laser printers. Every pixel is mapped, so these files are huge. Some kind of laser printer oriented hard copy drafting should be added to the tracking graphics, but the obvious choices so far do not blend very well with the menus and drawing primitives of our screen graphics.

\subsection{Symbols Options}

A limited number of plotting symbols can be provided by linking to a BNL MAD PIsymbol command. This format is used so the available choices can be easily customized, and also augmented. It also dodges dealing with the obscure and excessively complicated font mechanisms of vendor graphics systems, which are supposed to handle this kind of service. Symbol command formats are given in Section 5 below.

Plsymbol Name of a Plsymbol command to be linked for defining and drawing plotting symbols. Optional. If not given, single pixel points will be drawn.

Symbols(10) Optional list of names of plotting symbols, to be indexed by observation place.

Current choices are: $\mathbf{C R}=$ Cross, $\mathbf{P L}=$ Plus, $\mathbf{C I}=$ Circle, $\mathbf{S Q}=$ Square, $\mathbf{D I}=$ Diamond, $\mathbf{T R}=$ Triangle, $\mathbf{B a}=\mathrm{Bar}$, and $\mathbf{P T}=$ Point, a single pixel. The sequence will repeat for more than ten places. Default is Cross.

\section{FTrgen Command}

FTrgen is designed to generate sets of tracks related to phase ellipses around a space point, or around members of set of such points belonging to some spatial pattern. The ensemble of generated tracks are written to a data base module called a track bank. The simplest case uses the given phase coordinates of one space point to generate a set of tracks about the point. Using the emittances and initial amplitude functions, tracks are generated with $X$, Px coordinates corresponding to equally spaced angles about an $X$, Px plane emittance ellipse, and similarly for $Y$, Py coordinates from a Y, Py plane emittance ellipse. Alternatively, track points [X, Px, Y, Py, Ds, Dp] can be generated randomly within the separate $\mathrm{X}$ and $\mathrm{Y}$ 
emittance ellipses. In more elaborate cases involving more than one space point, various options permit generating a set of space points along the perimeters of a simple shape. A common pair of dimensions, width and height from the center to the farthest point, describes these simple shapes.

Only the first shape option encountered is cycled. Actually, for less than ten or so points, there isn't much difference among the various shapes. In a lattice without $X$ and $Y$ coupling, for some patterns $X$ and $Y$ values may repeat along a perimeter.

The FTrgen command should be inserted in a track command group after an FTrack track initialization command. A central track with all coordinates set to zero is written first into the track bank. The rest of the track bank is ordered as a set or group of tracks for each generating point [Xi, Yi]. Groups for the next point, and for all such points follow. This assembling of track groups is repeated for each value of the off momentum given. The completed track data has the overall format:

Track[ 6 Coordinates, ngtracks, npoints +1 , ndeltap ]

FTrgen attributes are:

Tracknam The name of the Track module to be produced. Useful if more than one set of tracks are to be generated by different generating commands. A default of *TRAC.01 will be assigned if no name is given.

Starts The name of a list of names of FStart commands, indicating that the starting space point(s) are to be taken from the given FStart initial track data, rather than from other options below.

Box Logical Flag: If True, space points will be taken from equally spaced intervals along the perimeter of a box shaped object. All of these optional shapes have a common half width of $D w$, and common half height of $D h$, centered at space point $X O, Y O$.

If there is no coupling, and phase patterns rather than space ones are being studied, the axes options are probably more useful than these shapes ones.

Cross Logical Flag: If True, space points will be taken from equally spaced intervals along a line $\mathrm{Y}=\mathrm{Y} 0$, parallel to the $\mathrm{X}$ axis, starting at $\mathrm{Xi}=\mathrm{X0}-\mathrm{Dw}$, and similarly along a line $\mathrm{X}=\mathrm{X} 0$, parallel to the $\mathrm{Y}$ axis, starting at $\mathrm{Yi}=\mathrm{Y} 0-\mathrm{Dh}$. At least four space points are needed.

Diamond Logical Flag: If True, space points will be taken at equally spaced intervals along the perimeter of a diamond shaped object.

Ellipse Logical Flag: If True, space points will be taken at equally spaced intervals along the perimeter of an ellipse shaped object.

Ring - Same as Ellipse, with equal axes.

Xaxis Logical Flag: If True, space points will be taken from equally spaced intervals along a line $\mathrm{Y}=\mathrm{Y} 0$, parallel to the $\mathrm{X}$ axis, starting at $\mathrm{Xi}=\mathrm{X} 0$ - Dw.

Yaxis Logical Flag: If True, space points will be taken from equally spaced intervals along a line $\mathrm{X}=\mathrm{X} 0$, parallel to the $\mathrm{Y}$ axis, starting at $\mathrm{Yi}=\mathrm{Y0}-\mathrm{Dh}$. Similar to Xaxis.

Dw Half width in meters along $X$ axis of given shape. Default is $.005 \mathrm{~m}$.

Dh Half height in meters along $Y$ axis of given shape. Default is $.005 \mathrm{~m}$.

Npoints Number of equally spaced points along perimeter of selected shape. A set of tracks defining a phase ellipse in $\mathrm{X}$ and in $\mathrm{Y}$ will be generated for each such point. Usually just enough points to define an outline or corners is sufficient, unless fine details are needed. Ignored for Starts option.

Random Logical Flag: If True, a group of tracks will be generated from random phase points inside of the initial phase ellipse, rather than as a set of equally spaced points on the perimeter of the phase ellipse.

Deltap(5) One or more off momentum values. The whole sequence of one or more ellipse groups of tracks is repeated for each value of Deltap given. 
Ngtracks The number of tracks in a group generated to define phase ellipses for each space point. Usually 10 or so is adequate.

$\mathrm{X} 0$, Y0, Px0, Py0

Coordinates of a basic starting track. If no shape is given, $\mathrm{X} 0$, $\mathrm{Px} 0$ will be the center of a phase ellipse in $\mathrm{X}, \mathrm{Px}$ coordinates, and $\mathrm{Y} 0, \mathrm{Py} 0$ will be the center of a phase ellipse in $\mathrm{Y}, \mathrm{Py}$ coordinates. If a shape is given, $\mathrm{X} 0$ and $\mathrm{YO}$ will be the center of a shape of size described by the $D w$ and $D h$ attributes. Space points $\mathrm{Xi}$, Yi on the perimeter or distributed within the shape together with $\mathrm{Px} 0$, Py0, will then be centers of $\mathrm{X}, \mathrm{Px}$ and $\mathrm{Y}, \mathrm{Py}$ phase ellipses used to generate groups of starting tracks.

/betax, /betay, /alfax, /alfay, /ex, /ey

Amplitude functions and emittances for the "closed" orbit at which starting tracks are being generated. These internal cells record initial orbit conditions entered on or produced by the FTrack command, and the emittances entered on a Beam command.

/ntrack Internal integer: total number of tracks produced by the generating command. This value will be the product of the number of points npoints by the number of tracks per point ngtracks, adjusted if needed, times the number of deltap values, plus a central first track with all coordinates zero.

/ngroups Internal integer: total number of track groups; equal to the number of generating points times the number of off momentum intervals.

Adefault(2) Internal name group pointing to default values for command.

/status, /stamp

\section{Symbols Command}

The Plsymbol command describes a few plot symbols, and offers a simple way to prescribe sizes and colors. For the present, it can be attached to the FTrplot and commands, but is easily extended. Dimensions in pixels are in real variables, so expressions can be used for scaling.

Attributes consist of a symbol dimension, and optional pen description group. Defaults are in parens following the prose.

Cross Full size of arm of cross symbol in pixels. (12.)

Crcolor(3) Integer color group, giving color index, line style, line width for cross. $(1=\operatorname{red}, 0,1)$

Plus Full size of arm of plus symbol in pixels. (10.)

Plcolor(3) Integer color group for plus symbol. ( $2=$ green, 0,1 )

Circle Radius of Circle symbol in pixels. (5.)

Cicolor(3) Integer color group for circle symbol. ( $4=$ blue, 0,1 )

Square Length of side of square symbol in pixels. ( 8 .)

Sqcolor(3) Integer color group for square symbol. ( 5 = magenta, 0,1 )

Diamond Length of side of diamond symbol in pixels. (10.)

Dicolor(3) Integer color group for diamond symbol. ( $6=$ cyan, 0,1 )

Triangle Length of side of triangle symbol in pixels. (10.)

Trcolor Integer color group for triangle symbol. $(8=$ grey, 0,1$)$

Bar Vertical length of bar symbol in pixels. (10.)

Bacolor Integer color group for bar symbol. ( $3=$ yellow, $0,4=$ width )

Ptcolor(1) Integer color for single point. (1 - red)

Setpen Logical Flag; if True, use color group integers from this Symbols command. Over rides current color set in program. (This use may interfere with label coloring).

Adefault(2) Internal name group pointing to module holding default values. 
/xpix, $\mathrm{y} / \mathrm{pix}$ Internal values for $\mathrm{x}, \mathrm{y}$ size of a pixel in user coordinates. Obtained in an initialization step after plotting is initialized and scales are set.

/status, /stamp

\section{Aperture Limits}

During tracking, besides dealing with given aperture limits on the specific collimator aperture types of lattice element, MAD checks orbit coordinates at every element against a crude limit of one meter. This crude limit may now be set to taste on the BNL MAD FRun tracking command by means of Xmax and $Y \max$ attributes:

$\mathrm{Xmax} \quad$ The maximum acceptable value of the $\mathrm{X}$ coordinate of a track in meters. For values above, the track is considered killed. The default is one meter.

$Y \max \quad$ Same for $Y$.

\section{Documentation Files}

Manual sources are in Unix troff (psroff) format.

Host: rapt.ags.bnl.gov

This Report: /usr/people/jn/Docum+/Trackplots.man

Plotfile: /usr/people/jn/Ags+/Trplots.call

\section{Example: AGS Tracking at Extraction}

1. A slightly off momentum particle is followed at three places in its orbit, prior to leaving the machine. The particle visits each arm of the pattern every three turns, moving outwards along the arm. The Single plot type graphics setup assigns different plot symbols and colors for the three observation points.

2. Three phase ellipses generated at three points, $\mathrm{X} 1=-.005, \mathrm{X} 2=0$, $\mathrm{X} 3=+.005$ are each drawn at three lattice points in an undisturbed AGS configuration, during the first turn. This is an example of the ellipse type of phase plot.

3. The same three phase ellipses are drawn at the same three lattice points, with the configuration set for ejection, during the first turn.

4. The same three phase ellipses are drawn at the same three lattice points, with the configuration set for ejection, during the sixth turn. 
\%

7.010.1. $=0$

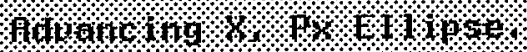

301.1.1.01:1.

10x.

$101,01000,0101001001010$

\subsection{0 .801}

11.

1.1010

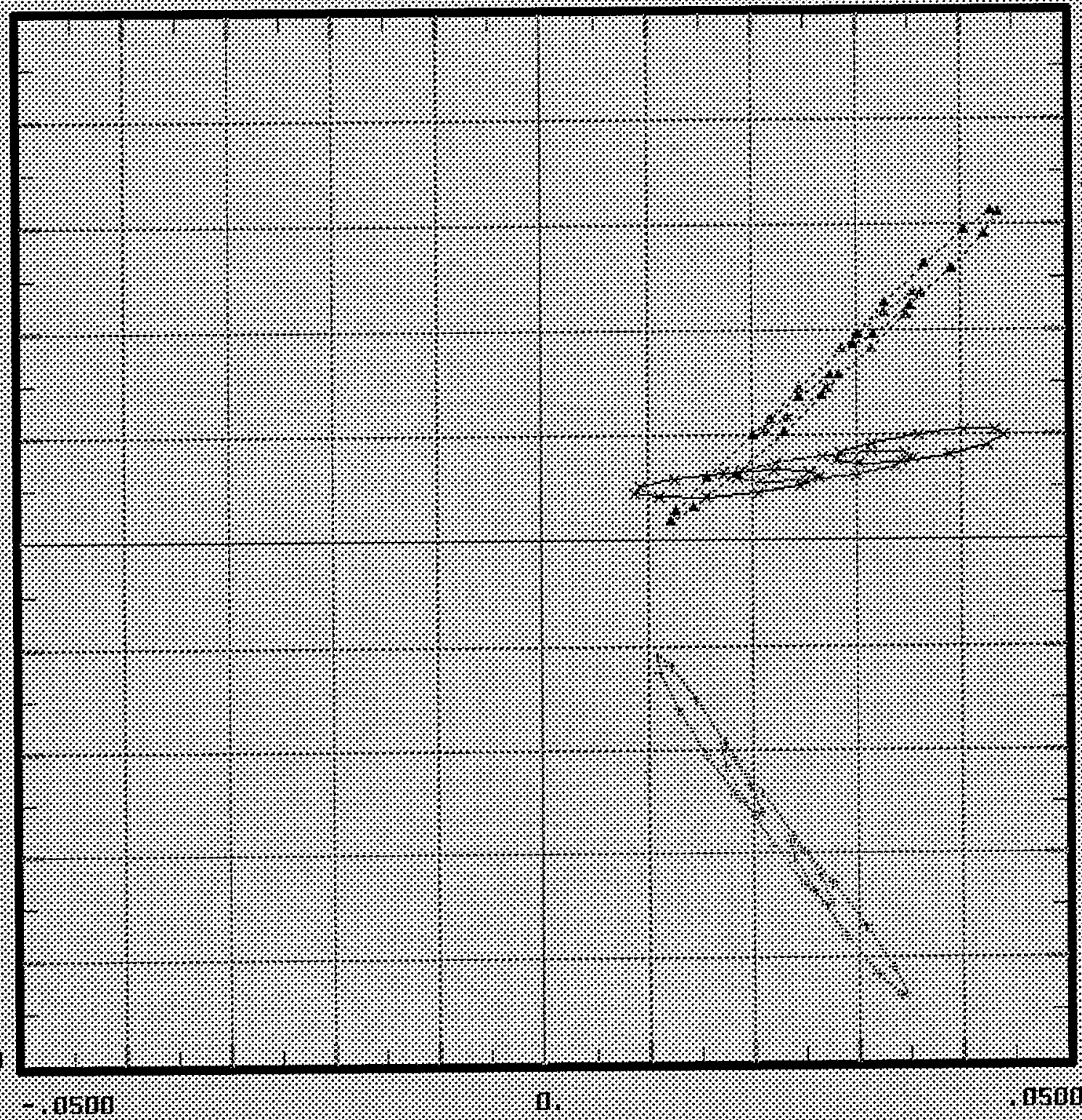

190.

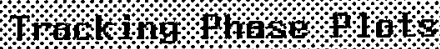

x

101.101010

1010101 
$-12-$



1016 .

61011010.

10

আ

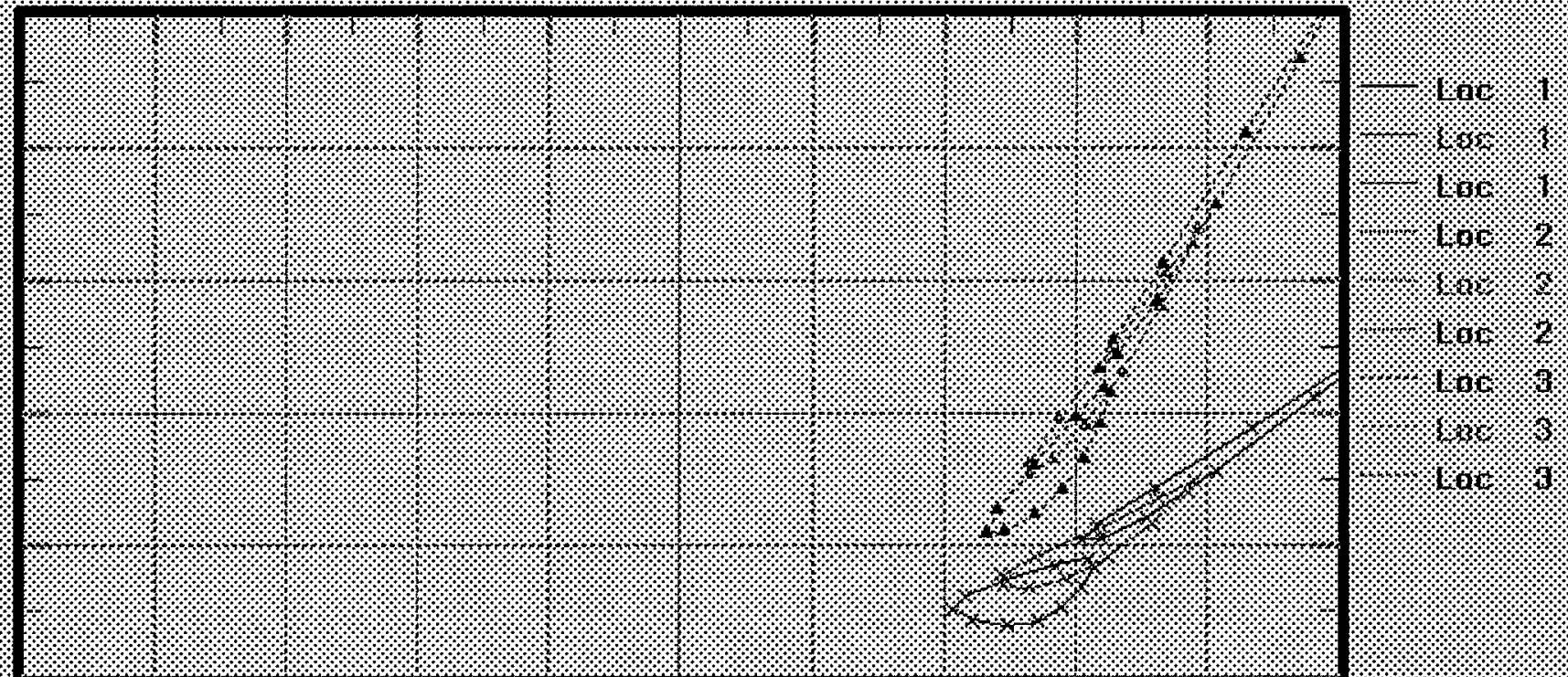



10.1019

19.

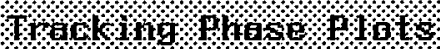

1601000101901011 